\title{
ABORDAJE MULTIDIMENSIONAL DEL SISTEMA SUELO DEL PERIURBANO, EN LA REGION SUR DE SANTA FE
}

\author{
Aradas Díaz, M.E. ${ }^{1}$; Benedetto, V. ${ }^{1}$; \\ Cardozo, F. ${ }^{1} ;$ Longo, A. ${ }^{1} \&$ TIMONI, R. ${ }^{1}$
}

\begin{abstract}
RESUMEN
El sistema suelo del periurbano, está cruzado por múltiples dimensiones y actores que complejizan su gestión. En la Plataforma de Innovación Territorial (PIT) del Instituto Nacional de Tecnología Agropecuaria (INTA) de Oliveros, se han identificado las dimensiones: económica/productiva, geográfica, ecológica y ambiental, organizativo-política, sociocultural, tecnológica e institucional. El objetivo de este trabajo fue profundizar el concepto y la gestión multidimensional del suelo del periurbano a través de la plataforma (PIT), vinculándolos a los objetivos institucionales del INTA. Metodológicamente se realizó un abordaje de sistemas complejos, con la descripción de los componentes/dimensiones y sus relaciones en el periurbano como unidad de análisis y la relación con los objetivos institucionales. Los resultados de la interacción entre las dimensiones permiten vincular conceptos como diversidad productiva, espacio crítico, territorio en disputa, diversidad de calidad y tipos de suelo, deficiencia en la gobernanza, heterogeneidad en el tamaño y formas de uso del suelo, tenencia de la tierra, leyes y ordenanzas de regulación, diversidad de actores involucrados, entre otras, que muestra un abordaje multidimensional, que a su vez se encuentran vinculados a los objetivos institucionales: competitividad, equidad y salud ambiental. El enfoque sistémico facilita la comprensión y dimensionamiento del sistema suelo del periurbano como espacio vivo donde sus propiedades emergen de las interacciones entre las dimensiones que lo componen.
\end{abstract}

Palabras clave: territorio, dimensiones, suelo, periurbano.

1.- EEA Inta Oliveros. Ruta Nacional 11, Km 353. (2206) Oliveros, provincia de Santa Fe. Tel: 03476 498010/011. Email: aradas.maria@inta.gob.ar Manuscrito recibido el 20 de mayo de 2020 y aceptado para su publicación el 5 de octubre de 2020.

Aradas Diaz, M.E.; Benedetto; V.; Cardozo, F.; Longo. A.; Timoni, R. Abordaje multidimensional del sistema suelo del periurbano, en la region sur de Santa Fe. FAVE - Ciencias Agrarias 19 (2): 11-24. CC BY-NC-SA 4.0 


\begin{abstract}
Multidimensional approach to peri-urban land system, in the southern region of Santa Fe.

Peri-urban land system is affected by multiple dimensions and actors that make its management complex. In the Territorial Innovation Platform of the INTA in Oliveros, the following dimensions have been identified: economic/productive, geographical, ecological and environmental, organizational-political, socio-cultural, technological and institutional. The aim of this paper is to deepen the concept and multidimensional management of the peri-urban soil, linking them to the institutional objectives. Methodologically, a description of each one of them is made, making a diagram that allows to visualize the interrelations between them. The results of the interaction between the dimensions make is possible to link concepts such as productive diversity, critical space, territory in dispute, diversity of quality and types of soil, governance deficiencies, heterogeneity in the size and methods of land use, land tenure, laws and regulations and diversity of actors involved, among others, imply a multidimensional approach, which, in turn, is linked to institutional objectives: competitiveness, equity and environmental health. The systemic approach facilitates the understanding and dimensioning of the peri-urban land as a living space where its properties emerge from the interactions between the dimensions that compose it.
\end{abstract}

Key words: territory, dimensions, land, peri-urband.

\section{INTRODUCCION}

El estudio del periurbano en la región sur de Santa Fe, está mediado por la ley 11.273. Dicha ley, que entre otros aspectos regula las aplicaciones de fitosanitarios, las distancias a las plantas urbanas, las formas de aplicación, y las condiciones para la aplicación. Si bien esta legislación es provincial, promueve la promulgación de ordenanzas a nivel local para la adecuación de las mismas a las condiciones de la comuna o municipio. Esto ha generado una gran diversidad de legislaciones a niveles locales que van dando marco a esta situación.

Por otro lado, los movimientos ecologistas y los ciudadanos-consumidores, están planteando la necesidad de producciones "más limpias" que aseguren alimentos para las comunidades desde la ruralidad, esta presión fue creciendo de modo que las comunas y municipios se vieron en la encrucijada no solo de regular las aplicaciones sino de considerar el pedido de producciones sostenibles. Además, la escasa planificación urbana dificulta la delimitación de esta área, por lo que se da, el avance de las ciudades sobre las áreas rurales, sin suficientes regulaciones.

Las tensiones generadas en los territorios dan motivo a esta investigación. En los últimos años se vienen contrastando las percepciones que poseen los sectores urbanos y rurales en relación a los efectos e incidencias de la aplicación de productos agroquímicos utilizados en la producción agropecuaria generándose diferentes conflictos que son abordados con reglamentaciones 
locales a través de ordenanzas municipales y comunales que plantean, a partir de la demanda social sobre la forma de producir y la protección del ambiente, restricciones en la aplicación de productos agroquímicos en áreas cercanas a los centros poblados y escuelas o instituciones rurales.

El objetivo de este trabajo es profundizar el concepto y la gestión multidimensional del suelo del periurbano a través de la Plataforma de Innovación Territorial (PIT), vinculándolos a los objetivos institucionales del INTA. Se realiza en el marco de la PIT: Innovación tecnológica y organizacional para el abordaje del territorio urbano-rural del INTA Oliveros.

\section{ANTECEDENTES}

El gobierno de la provincia de Santa Fe viene implementando diferentes estrategias de abordaje de la producción agropecuaria en el área del periurbano, problemática que se vincula a otros intereses sobre el uso de ese suelo, como son el industrial y el inmobiliario, entre otros.

Para la regulación de la producción agropecuaria se realizó el lanzamiento del Programa de Buenas Prácticas Agropecuarias (B.P.A.) que se focaliza en la promoción de sistemas eficaces de gestión y tiene como objetivo "lograr el cumplimiento efectivo de la Ley de Productos Fitosanitarios y la de Conservación y Manejo de Suelos". Por el otro, desde 2017 se encontraba en funcionamiento el "Programa de producción sustentable de alimentos periurbanos" ${ }^{1}$ que busca promover y fortalecer alternativas a la producción convencional, considerando y repensando los aspectos ambientales, sociales y económicos.

A su vez el Ente de Coordinación Metropolitana (ECOM) ${ }^{2}$ que agrupa a algunas localidades del Sur de la provincia de Santa $\mathrm{Fe}$, ha generado un "Informe Eje Temático Sectorial Periurbano y Rural” en el cual se incluyen diagnósticos y clasificación de periurbanos; propuesta de ordenamiento territorial para cada una de las localidades y propuesta productiva apropiada a cada tipología de periurbano. Además, la Municipalidad de Rosario viene implementando el "Programa Alimentario-Cinturón Verde", al igual que otras localidades de la provincia que tienen propuestas y acciones en el periurbano y lo abordan en forma puntual.

Por otra parte, el INTA Estación Experimental Agropecuaria Oliveros, en el área de estudio (Figura 1), recibe constantemente la demanda de los gobiernos locales, de productores, de vecinos, sobre el abordaje de la producción.

Para lo cual la institución genera diversas líneas en este sentido: acciones de desarrollo local, como por ejemplo Oliveros en Red -Premiado por FAO como innovación de abordaje territorial-(Aradas Diaz et al., 2018), Godoy (Aradas Diaz, 2017), Las Rosas, Tortugas, Roldan-“mesa de dialogo interactoral" como un dispositivo para la gobernanza-, Casilda, entre otras, acompañamiento en lo normativo en las localidades como Arequito, Oliveros, San Genaro (Propersi et al., 2017); propuestas producti-

1.- Programa de producción sustentable de alimentos en periurbanos

https://www.santafe.gov.ar/index.php/web/content/view/full/215582/(subtema)/112065

2.- Ente de Coordinación Metropolitana de Rosario. https://www.rosario.gob.ar/web/gobierno/empre-

sas-y-organismos-del-estado/ente-de-coordinacion-metropolitana-ecom

Revista FAVE - Ciencias Agrarias 19 (2) 2020 


\section{E. Aradas Díaz et al.}

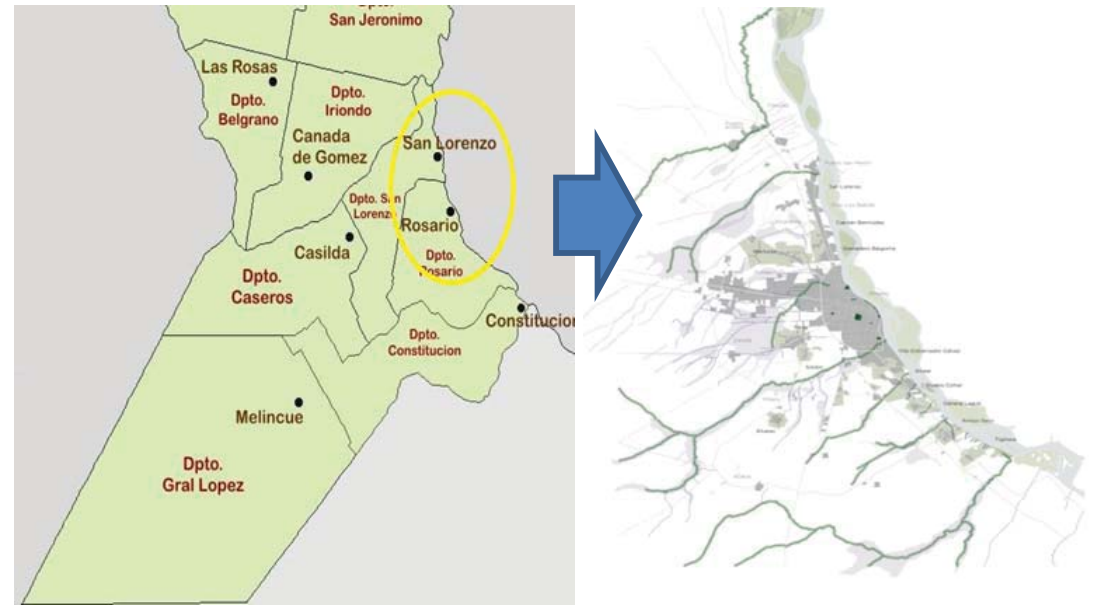

Figura 1: Área de estudio.

Figure 1: Study area.

vas para distintas tipologías del periurbano -ECOM- (Longo et al., 2018); experiencias comunicacionales (Totoras) (Fior y Guglielmone, 2017) y tecnologías adaptadas a las situaciones de las comunas y municipios, según cada caso. Ejemplos de esto último son: el desarrollo del algoritmo de gestión del periurbano INTA-AUDEAS-CONADEV (D’Angelo et al., 2018); generación de tecnología de aplicación de fitosanitarios con baja deriva (Carrancio et al., 2016); modelos de bordes forestales multiestrato (Cardozo, 2018), modulo experimental y demostrativo agroecológico agrícola ganadero (Benedetto et al., 2018), validación de maquinaria apropiada a sistemas de no aplicación de agroquímicos. También se realiza el asesoramiento y acompañamiento para la gestión de procesos productivos que incluyen las BPA y la producción agroecológica de alimentos, el agregado de valor en origen $^{3}$ y la comercialización en mercados de cercanía que contribuyen al desarrollo local (Benedetto et al., 2019). Se han generado espacios de articulación y reflexión con Ministerio de la Producción, Ministerio de Medio Ambiente, Colegio de Ingenieros, Universidades, Comunas y Municipios (Aradas Diaz et al., 2019) y numerosas instancias de capacitación con la participación de más de 2.000 actores.

Sin embargo, es importante destacar que muchas de estas acciones se encuentran dispersas y con escasa interacción entre ellas ${ }^{4}$.

\section{MARCO DE ANÁLISIS}

El espacio periurbano es un espacio geográfico entre el campo y la ciudad, constituye un "continuum” que transforma el limite rural urbano en un concepto integrado de territorio, este espacio tiene características y actores propios que lo hacen objeto de estudios, investigaciones e inter-

3.- como la experiencia de harina de trigo agroecológico en convenio público privado

4.- Documento Base PIT: Innovación tecnológica y organizacional para el abordaje del territorio urbano-rural. https://inta.gob.ar/documentos/un-lugar-para-debatir-y-trabajar-en-los-periurbanos 
venciones particulares, en él pueden existir elementos y procesos de la ruralidad tanto como de la urbanidad que le dan su particular identidad. Podría además contribuir a la producción local adaptada a la demanda de consumo de la región, potenciando las oportunidades de trabajo.

Morello (2000) define al sistema periurbano como un espacio muy dinámico y complejo que tiene todas las características de una interface ecológica y de una frontera socio-productiva, que no es campo, ni tampoco ciudad.

En el suelo periurbano coexisten distintos elementos y actores que resultan en un paisaje particular con dinámicas propias: la naturaleza (topografía, vientos, cursos de agua, flora y fauna), los sistemas productivos variados (especialmente la producción rural extensiva e intensiva), las infraestructuras y los espacios habitacionales y/o productivos (viviendas rurales y urbanas o loteos deslindados de las plantas urbanas, establecimientos productivos rurales, escuelas, centros recreativos, áreas protegidas). Los actores tienen intereses, percepciones y agendas divergentes, lo que sumado a la falta de ordenamiento territorial, generan en su interacción tensiones y conflictos de complejo abordaje. Los gobiernos locales son un actor clave en la gestión y reorganización de este espacio.

La dinámica y la heterogeneidad de este espacio en permanente transformación hace necesario un abordaje de los problemas y oportunidades como sistema complejo para establecer una propuesta de desarrollo generando en este espacio intermedio un nuevo modo de relación urbano rural.

Las demandas y características del territorio urbano rural, sus problemáticas y la priorización institucional, hacen necesario el desarrollo de una plataforma específica que articule propuestas y genere sinergias entre los diferentes actores.

El marco de análisis de este trabajo, lo constituyen la herramienta programática plataforma de innovación territorial y los tres objetivos institucionales.

El desafío planteado requiere compatibilizar los objetivos institucionales de competitividad, salud ambiental y equidad social, enmarcándolos en una estrategia de desarrollo equilibrada y sustentable en el largo plazo para superar las desigualdades territoriales.

Competitividad sistémica: Es el proceso de construcción de ventajas competitivas dinámicas. Contempla una relación explícita entre eficiencia, productividad, cuidado del ambiente y el mejoramiento de la calidad de vida de la comunidad y en los territorios. Es el producto de una interacción compleja y dinámica entre el Estado, las empresas, las instituciones intermedias y la capacidad organizativa de la sociedad.

Salud ambiental: El concepto comprende los aspectos de la salud humana, incluida la calidad de vida y el bienestar social, que son determinados por factores ambientales.

Equidad: El concepto promueve la igualdad de condiciones y oportunidades para todas las personas, como también la distribución justa de la riqueza entre los miembros de una sociedad. Las políticas de equidad social se refieren al acceso a los servicios públicos de salud, de educación y de infraestructura.

El propósito de las PIT consiste en facilitar la implementación de formas de organización y gestión conjuntamente con otras instituciones y actores del medio, promoviendo el desarrollo de productos y procesos que viabilicen la resolución de los pro- 
blemas de crecimiento e inclusión social de cada región.

El punto de partida de dicha plataforma, radica en establecer consensos que permitan instaurar los alcances de cada PIT, tomando en consideración un enfoque multidimensional, que incluya no sólo los aspectos económico-productivos, sino también elementos ambientales, tecnológicos, político-administrativos, sociales y culturales.

El funcionamiento de la plataforma se propone en varias fases operativas: una primera fase dónde se realiza la incorporación de integrantes la que estará condicionada por el interés que despierte la iniciativa y el compromiso de los mismos para la innovación. También en esta fase se debe iniciar el proceso participativo de identificación de problemas y oportunidades. Es relevante el rol del INTA para facilitar el proceso.

Una segunda fase para facilitar la organización de la demanda y priorización de problemas y oportunidades, así cómo, proyectar la factibilidad de su abordaje a través de los proyectos. La construcción de redes de innovación. Y una tercera fase dónde se espera que las organizaciones e instituciones se “apropien” de la Plataforma, llevando adelante el seguimiento de las acciones priorizadas y el mantenimiento de las redes para la innovación.

En cuanto a la organización y estrategia de la misma se tendrán cuatro niveles representados en la Figura 2:

1-Equipo de Gestión Interna Operativa:

- Coordinador de la PIT

- Técnicos de INTA participantes del equipo de gestión operativa

- Extra INTA que participan del equipo de gestión operativa.

2- Miembros de la Plataforma:
- De INTA: Coordinador PIT, Director EAA, Coordinadores de Área de Desarrollo y de Investigación, Representantes CLA EEA y/o AER.

- Públicos: Gobiernos (Provincia, Municipios, Comunas, etc.): Representantes de Ministerios Provinciales (Producción, Medio Ambiente, Educación, Salud, Desarrollo Social, Trabajo y Seguridad Social, Ciencia Tecnología e Innovación Productiva, Gobierno, Justicia y Derechos Humanos, etc.), ASSAL (Agencia Santafecina de Seguridad Alimentaria); Representantes de los poderes Legislativo y Judicial; de las áreas de Educación, Salud, así como de la Universidad Nacional de Rosario y de la Universidad Tecnológica Nacional, Escuelas Agrarias y Técnicas.

- Privados: Universidades, organizaciones de productores, organizaciones de la sociedad civil (consumidores, ecologistas, productores, vecinales), Cámaras empresariales (de aplicadores, de acopiadores, de emprendimientos turísticos, de inmobiliarias, etc.) Colegios Profesionales, certificadores, Cooperativas.

3-Comisiones temáticas Ad hoc:

- Referentes temáticos de INTA y extraINTA, que abordan temáticas especificas acordadas.

4- Nodos locales:

- Están conformados por los miembros locales de INTA y de las instituciones y actores de las localidades vinculadas al periurbano del centro y sur de Santa Fe y que expresen la necesidad de abordaje de la problemática periurbana y su articulación con la plataforma. Serán promovidos desde las agencias de INTA como ámbitos de trabajo conjunto.

\section{METODOLOGIA}




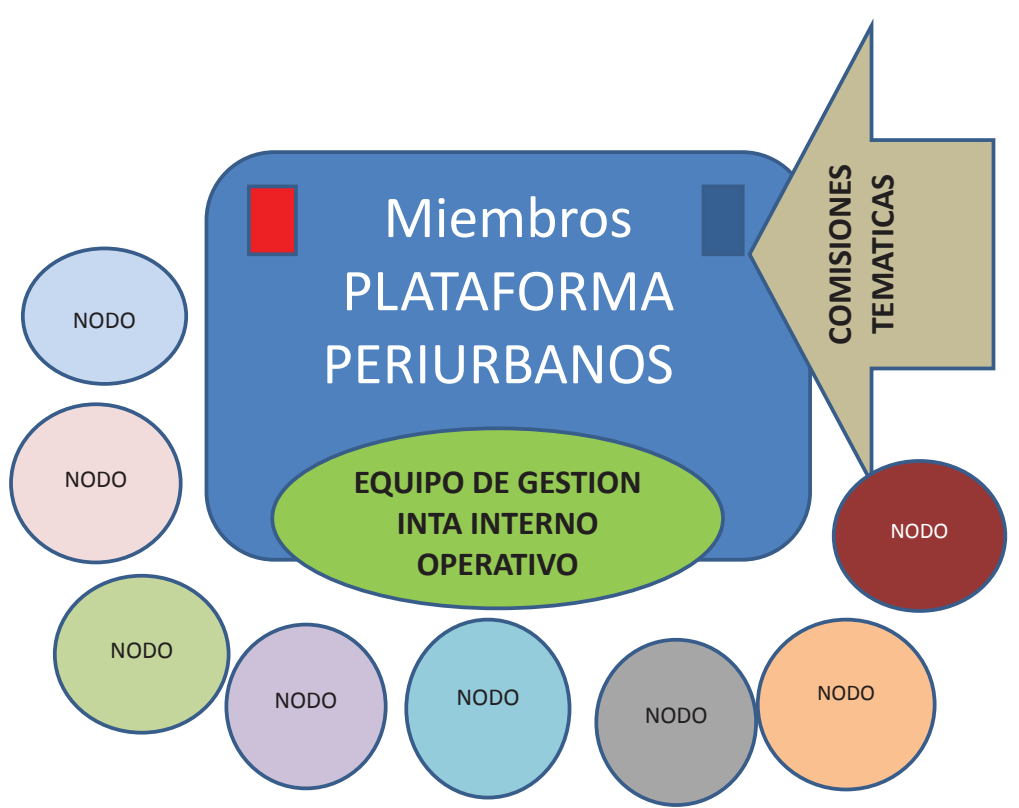

Figura 2: Estructura y gestión de la PIT INTA Oliveros.

Figure 2: Structure and management of the PIT INTA Oliveros.

Fuente: Elaboración Propia.

Source: Self made.

Metodológicamente se realizó un abor- relato explicativo de cada una de ellas y se daje de sistemas complejos, siguiendo a propuso un esquema interpretativo de relaRolando García (2006), para lo cual se es- ciones posibles (interacciones) del sistema. tableció los límites del sistema, sus com- Posteriormente, a esta sistematización por ponentes y se avanzó en la estructura de dimensiones/componentes, se la analizó relaciones entre ellos. Los resultados de mediante una matriz que la vincula con los talleres con actores claves, fueron las he- objetivos institucionales: competitividad, rramientas utilizada y se organizaron en equidad y salud ambiental.

seis dimensiones (1-económica productiva; Considerando la heterogeneidad de los 2- geográfica, ecológica y ambiental; 3- or- periurbanos (tamaño de la localidad, cercaganizativa y política; 4- social y cultural; nía a los centros urbanos más grandes, vías 5- institucional; 6-tecnológica) del sistema de comunicación, etc.) la descripción de las suelo del periurbano. En cada una de las dimensiones se realiza en forma genérica dimensiones (componentes) se realizó un buscando contemplar los elementos comu- 
nes. Desde el Centro Regional Santa Fe ${ }^{5}$, se ha trabajado en la definición de seis dimensiones básicas para pensar y comprender las dinámicas territoriales, en este caso en el periurbano. Las mismas son las utilizadas en el presente trabajo para el análisis:

- Económica/productiva: Incluye los diferentes sistemas/cadenas de producción y tipos de empresas predominantes, así como las finanzas, los medios de producción, las instalaciones, infraestructuras y la dotación de servicios de apoyo a la producción disponibles en un territorio.

- Geográfica, ecológica y ambiental: Constituye una dimensión central y diferenciadora de cada territorio, que contempla las condiciones agroecológicas del medio, su paisaje, fuentes de materias primas, agua, energía, así como los bienes y servicios medioambientales que el medio natural ofrece.

- Organizativa y política: Se tomarán en consideración las redes de instituciones presentes en cada territorio, así como también las formas de Gobierno que delimitan las regiones. La expresión política de formas de Gobierno alude a las diversas maneras de organización de un estado o el ejercicio del poder. En el caso de Santa Fe, la forma de organización adoptada en el territorio provincial se distribuye en 19 departamentos, 365 distritos, 352 municipios y comunas.

- Social y cultural: Representada por la distribución poblacional y el conjunto de normas y valores que rigen las interacciones entre las personas. La premisa básica es colaborar a la construcción y el desarrollo de las comunidades, involucrando a todas las partes interesadas, reforzando las redes sociales y fomentando el arraigo territorial.
- Institucional: Una delimitación a considerar para el establecimiento de las PIT se encuentra constituida por la estructura organizativa y administrativa del INTA, tanto a nivel nacional, como regional. En la provincia de Santa Fe, la institución desarrolla sus actividades por medio de tres estaciones experimentales agropecuarias (EEA), cada una de ellas con un perfil de actividad claramente definido. Complementan la estructura institucional un conjunto de 22 agencias de extensión rural (AER) que se vinculan con la gran diversidad de actores territoriales.

- Tecnológica: La dimensión tecnológica se considera relevante puesto que motoriza la productividad y competitividad de los sistemas y las cadenas productivas. Se incluyen aquí las tecnologías, herramientas, modos de gestión y equipamientos utilizados en las producciones predominantes.

El espacio dónde se generaron los insumos de este trabajo fue en una Jornada - Taller: Alternativas productivas en el periurbano, espacio de intercambio y análisis de propuestas realizada el 9 de Octubre del 2019. Para dar cuenta de su magnitud, compartimos las características de los participantes y las provincias representadas. En cuanto a los participantes: Se logró un total de 172 participantes del encuentro. De los asistentes un 22\% fue de productores agropecuarios, representantes de cooperativa y organizaciones del sector; 26\% del INTA y la Secretaría de Agricultura Familiar de las provincias del centro del país; un 21,5\% de universidades de la región; el 10,5 \% del Colegio de Ingenieros Agrónomos y Asesores Técnicos; el 6,4 \% de Comunas y Municipios; el 5,2 \% de organismos del estado provincial y el $8 \%$ de particulares. 
La convocatoria permitió la presencia de muchos representantes de la provincia de Santa Fe y de las Provincias de Córdoba, Entre Ríos y Buenos Aires, lo que demuestra la vigencia y la necesidad del abordaje de la temática de periurbanos en la región pampeana. (Informe no publicado de la Jornada Taller- Cardozo, F.)

\section{RESULTADOS}

Los resultados se generaron en cuatro etapas: las dos primeras fueron en el marco de la Jornada- taller y las otras dos son etapas de análisis del equipo técnico, autores de este trabajo.

Etapa I- Se trabajó en grupos donde se aplicó la técnica de brainstorming listado problemas que se encuentran en el territorio del periurbano.

Etapa II- Los problemas se ordenaron para caracterizar cada dimensión, dónde quedó planteado una aproximación diagnóstica multiactoral y multisectorial, como producto del taller.

\section{- Económica/productiva:}

1- Escaso desarrollo de mercado de cercanías.

2- Dificultades para agregar valor en origen.

3- Aumento de los costos de producción,

4- Falta de sistemas de garantías del producto obtenido en estas zonas.

5- Simplificación del modelo productivo y escasa diversificación.

6- Falta de propuestas productivas que contemplen la armonización con los intereses de la sociedad local.
- Geográfica, ecológica y ambiental:

1- Degradación de recursos naturales.

2- Pérdida de biodiversidad.

3- Contaminación ambiental.

4- Adaptación y mitigación del cambio climático.

- Organizativa y política:

1- Falta de visión consensuada del desarrollo a mediano y largo plazo.

2- Heterogeneidad de normativas locales.

3- Precariedad en la tenencia de la tierra.

4- Falta de ordenamiento del territorio.

\section{- Social y cultural:}

1- Proceso de urbanización desordenada en ciudades intermedias.

2- Urbes rurales muy dependientes del sistema agrario.

3- Degradación del entramado social.

4- Falta de fuentes de trabajo local.

\section{- Institucional:}

1- Baja vinculación interinstitucional público-privada.

2- Escasos espacios de participación y reflexión.

3- Escasa integración de la producción-agregado de valor-comercialización a nivel local.

\section{- Tecnológica:}

1- Escasos procesos socio-productivos donde se minimicen riesgos ambientales que permitan un desarrollo armónico e integrado de los diferentes sectores.

2- Falta de tecnología apropiada para posibilitar la convivencia y el desarrollo de los diferentes actores.

3- Uso de tecnologías inadecuadas para las necesidades del territorio. 
4- Tecnología de manejo convencional en uso en los sectores periurbanos.

5- Escasas tecnologías de procesos, de insumos y de maquinarias apropiadas al periurbano.

Etapa III: Análisis de las relaciones entre las dimensiones, posteriormente lo continúo el equipo técnico, con la lógica del análisis del Stockholm Resilience Centre (Pretlove y Blasiak, 2018).

\section{Relaciones entre las dimensiones:}

La problematización del sistema complejo del periurbano, produce un conocimiento que pretende poner en dialogo dimensiones muy distintas, con formas de cuantificación diferente, por lo que el estudio de la unidad sistémica se torna en un conocimiento situado y específico. Hemos ordenado en la Figura 3 las dimensiones siguiendo la lógica del análisis del Stockholm Resilience Centre (Pretlove y Blasiak, 2018), siendo la base del mismo la geográfica, ecológica y ambiental, como una dimensión preexistente a las anteriores, y que a su vez hace de soporte a la dimensión socio cultural, y esta última a la económico -productivo. Entendiendo que el soporte natural es ocupado por una sociedad que se organiza a través de la economía.

Estas tres dimensiones se ven relacionadas unas a otras de forma vertical, generando relaciones funcionales entre ellas, de modo que cuando se produce un desequilibrio en cualquiera de las tres; las mismas se ven afectadas porque la interrelación es de caraterístias físicas, de ocupación del espacio, de las formas de producción y consumo.

Las interrelaciones son tales y en ambos sentidos, que cambios en cualquiera de ellas produce un efecto en las demas. El tipo de efecto que produzca tiene que ver, por ejemplo, con un cambio en el tamaño o intensificación de una actividad productiva, que produce externalidades como desechos o degradación del ecosistema, afectando el suelo del periurbano, la calidad de vida y la dinámica de la economía local. Como se puede ver en la Figura 3 donde se interconectan los Objetivos de Desarrollo Sostenible, y a su vez que se organizaron por los ejes de ambiente, sociedad y economía, para poder explicar la complejidad del desarrollo sostenible y que el tamaño de las dimensiones que lo componen son diferentes.

Las otras tres dimensiones tecnológica, organizativo-política e institucional (con eje en esta última) atraviesa a las primeras tres (ambiental-social-económica), donde la dimensión institucional aparece como un eje ordenador como es el caso del ODS 17 “alianzas para lograr los objetivos” por lo que la institucionalidad y las formas organizativas, que estructuran a la sociedad son fundamentales para la generación de alianzas, para generar procesos de desarrollo desde sus distintos actores público-privados.

La tecnología se presenta como una dimensión importante en cuanto facilitadora de procesos que permiten mantener estrategias ante los cambios propios de la sustentabilidad, con las tres primeras dimensiones. Está vinculada fuertemente a los procesos institucionales de modo que éstos se empoderan de las tecnologías para mejorar las relaciones entre ambiente-sociedad y economía.

En cuanto a la dimensión organizativo-política, es la que tiene que sumar la visión prospectiva y las relaciones del espacio local con las otras escalas de manera de operativizar políticas que permitan cuidar estas relaciones de sostenibilidad. 


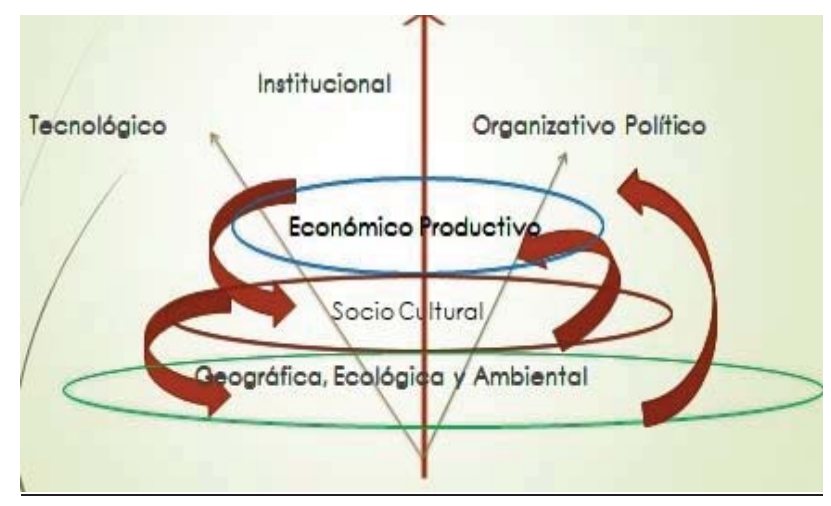

Figura 3: Relaciones entre las dimensiones.

Figure 3: Relationships between dimensions.

Fuente: Elaboración Propia.

Source: Self made.

En el abordaje multidimensional del suelo del periurbano, desde el punto de vista agropecuario, encontramos:

Entre las dimensiones ambiental, económica, mediada por la tecnológica, emerge la diversidad productiva por la heterogeneidad en el tamaño y formas de uso del suelo.

Vinculando a las dimensiones social y económica, se visualiza un espacio crítico y territorio en disputa mediado por la diversidad de calidad y tipos de suelo e intereses sectoriales.

La interacción entre las dimensiones políticas, institucionales y sociales, permiten encontrar deficiencia en la gobernanza, tenencia de la tierra, leyes y ordenanzas de regulación y diversidad de actores involucrados.

Etapa IV: Análisis de las relaciones entre las dimensiones/elementos del sistema y los objetivos institucionales.
En la Tabla 1 observamos que las principales problemáticas a tratar en cada una de las dimensiones, son atravesadas por los tres objetivos institucionales, dando cuenta de que la mirada institucional se torna estratégica en la medida que pueda encontrar formas de abordar integralmente los territorios, en este caso el periurbano.

Los resultados son coincidentes con otros trabajos que dan cuenta de que "la interpretación del periurbano desde la perspectiva de los sistemas complejos permite realizar un análisis integrado del territorio teniendo en cuenta los atributos considerados relevantes para caracterizar los procesos que se dan en estas áreas de interfase (Zulaica y Ferrero, 2011) y donde "la teoría de los sistemas complejos de Rolando García nos provee una perspectiva multiescalar y multidimensional” (Fernández Equiza, 2017). 
M. E. Aradas Díaz et al.

Tabla 1: Vinculaciones entre las Dimensiones y los Objetivos Institucionales del INTA Table 1: Links between Dimensions and Institutional Objectives of INTA.

Fuente: Elaboración Propia.

Source: Self made.

\begin{tabular}{|c|c|c|c|}
\hline $\begin{array}{l}\text { Objetivos } \\
\text { Institucionales/Dimensiones }\end{array}$ & Competitividad & Equidad & Salud Ambiental \\
\hline $\begin{array}{l}\text { Geografía, ecológica y } \\
\text { ambiental }\end{array}$ & $\begin{array}{l}\text { Pérdida de } \\
\text { Biodiversidad. } \\
\text { Contaminación } \\
\text { Ambiental } \\
\end{array}$ & $\begin{array}{l}\text { Degradación de } \\
\text { recursos naturales }\end{array}$ & $\begin{array}{l}\text { Adaptación y } \\
\text { mitigación al } \\
\text { cambio climático. }\end{array}$ \\
\hline Socio Cultural & $\begin{array}{l}\text { Urbes rurales muy } \\
\text { dependientes del } \\
\text { Sistema Agrario }\end{array}$ & $\begin{array}{l}\text { Degradación del } \\
\text { entramado social. } \\
\text { Falta de fuentes de } \\
\text { trabajo local }\end{array}$ & $\begin{array}{l}\text { Proceso de } \\
\text { urbanización } \\
\text { desordenado en } \\
\text { ciudades } \\
\text { intermedias }\end{array}$ \\
\hline Económico Productivo & $\begin{array}{l}\text { Dificultades para } \\
\text { agregar valor en } \\
\text { origen. Aumento de los } \\
\text { costos de producción. } \\
\text { Falta de sistemas de } \\
\text { garantías del producto } \\
\text { obtenido en esa zona. }\end{array}$ & $\begin{array}{l}\text { Escaso desarrollo de } \\
\text { mercados de cercanía. } \\
\text { Falta de propuestas } \\
\text { productivas que } \\
\text { contemplen la } \\
\text { armonización con los } \\
\text { intereses de la } \\
\text { sociedad local }\end{array}$ & $\begin{array}{l}\text { Simplificación del } \\
\text { modelo productivo } \\
\text { y escasa } \\
\text { diversificación. }\end{array}$ \\
\hline Institucional & $\begin{array}{l}\text { Escasa integración de } \\
\text { la producción, valor } \\
\text { agregado y } \\
\text { comercialización }\end{array}$ & $\begin{array}{l}\text { Escasos espacios de } \\
\text { participación y } \\
\text { reflexión }\end{array}$ & $\begin{array}{l}\text { Baja vinculación } \\
\text { interinstitucional } \\
\text { público -privada. }\end{array}$ \\
\hline Tecnológica & $\begin{array}{l}\text { Uso de tecnologías } \\
\text { inadecuadas para las } \\
\text { necesidades del } \\
\text { territorio. Tecnología } \\
\text { de manejo } \\
\text { convencional en uso } \\
\text { en los sectores } \\
\text { periurbanos. } \\
\text { Escasas tecnologías } \\
\text { de procesos, de } \\
\text { insumos y de } \\
\text { maquinarias } \\
\text { apropiadas al } \\
\text { periurbano }\end{array}$ & $\begin{array}{l}\text { Escasos procesos } \\
\text { socio-productivos } \\
\text { donde se minimicen } \\
\text { riesgos ambientales } \\
\text { que permitan un } \\
\text { desarrollo armónico e } \\
\text { integrado de los } \\
\text { diferentes sectores. }\end{array}$ & $\begin{array}{l}\text { Falta de } \\
\text { tecnología } \\
\text { apropiada para } \\
\text { posibilitar la } \\
\text { convivencia y el } \\
\text { desarrollo de los } \\
\text { diferentes actores. }\end{array}$ \\
\hline Organizativa política & $\begin{array}{l}\text { Falta de visión } \\
\text { consensuada del } \\
\text { desarrollo a mediano y } \\
\text { largo plazo }\end{array}$ & $\begin{array}{l}\text { Heterogeneidad de } \\
\text { normativas locales. } \\
\text { Precariedad en la } \\
\text { tenencia de la tierra. }\end{array}$ & $\begin{array}{l}\text { Falta de } \\
\text { ordenamiento del } \\
\text { territorio. }\end{array}$ \\
\hline
\end{tabular}




\section{CONCLUSIONES}

El enfoque sistémico permite la comprensión y dimensionamiento del sistema suelo del periurbano como espacio vivo donde sus propie $\neg$ dades emergen de las interacciones entre sus dimensiones (ecológica, social, económica, tecnológica, política e institucional).

El suelo de este espacio esta cruzado por múltiples dimensiones y actores que complejizan su gestión. Por lo que se organizaron las problemáticas en seis dimensiones: económica/productiva, geográfica, ecológica y ambiental, organizativo-política, sociocultural, tecnológica e institucional, para comprender la complejidad y generar un dispositivo de abordaje que permita considerar las relaciones de interdependencia, donde se puede observar las implicancias de unas sobre otras y de donde surgen emergentes que no son explicadas por cada dimensión en particular sino por sus interacciones.

Los cuatro niveles de organización de la PIT (equipo de gestión, miembros, mesas temáticas y nodos locales) entendemos que facilitarán el abordaje que integre los objetivos institucionales y las dimensiones en la complejidad del periurbano. Con el desafío de incorporar nuevos conceptos y estrategias como paisajes alimentarios, circuitos cortos, círculos virtuosos, innovación social, democracia alimentaria, procesos de gobernanza, vínculo urbano-rural, gobernanza alimentaria, justicia ambiental, perspectivas críticas, entre otras.

\section{BIBLIOGRAFIA}

Aradas Diaz ME, Benedetto M, Cardozo F, Longo A, Timoni R. 2018. Avances de procesos territoriales en transición agroecológica en el periurbano de la localidad de Oliveros Santa Fe. En: Titonell P y Giobellina B, Periurbanos hacia el Consenso. Libro 1. Ediciones INTA, 674 pp. https://repositorio.inta.gob.ar/ xmlui/handle/20.500.12123/3035

Aradas Diaz ME, Benedetto M, Cardozo F, Longo A, Timoni R. 2019 Abordajes del Periurbano: Espacio dinámico y complejo. ELER 2019 Primer Encuentro Latinoamericano de Estudios del Rururbano 7 y 8 de Marzo, Santa Fe Argentina.

Aradas Diaz, ME. 2017. Desarrollo Territorial a escala local en el sur de Santa Fe. En: Milo Vaccaro M y Aradas Diaz ME. Revista Construyendo Vínculos 2. Ediciones INTA EEA OLIVEROS. 120 pp. https://inta.gob.ar/sites/ default/files/inta.revista-construyendo-vinculos.pdf

Benedetto V, Gamundi JC, Timoni R, Cardozo F, Longo A, Guglielmone P, Aradas Díaz ME. 2018. Módulo experimental en transición agroecológica. Revista para Mejorar la producción $\mathrm{N}^{\circ}$ 56. Ediciones INTA EEA OLIVEROS. https://inta.gob.ar/documentos/modulo-experimental-en-transicion-agroecologica

Benedetto V, Cardozo F, Longo A, Timoni R, Aradas Diaz ME. 2019. Agroecología y Desarrollo. ELER 2019 Primer Encuentro Latinoamericano de Estudios del Rururbano 7 y 8 de Marzo, Santa Fe Argentina.

Cardozo F. 2018. Bordos Urbano Rurales BUR- "Cortinas forestales multiestrato multipropósito” Ing. Forestal Magister INTA EEA Oliveros. En: Titonell P y Giobellina B, Periurbanos hacia el Consenso. Libro 1. Ediciones INTA, 674 pp. https://repositorio. inta.gob.ar/xmlui/handle/20.500.12123/3035 
M. E. Aradas Díaz et al.

Carrancio L, Massaro R, Cardozo FV. 2016. Criterios para el uso de plaguicidas en áreas críticas. Cartilla Técnica INTA. https://inta.gob. ar/documentos/criterios-para-el-uso-de-plaguicidas-en-areas-criticas

D’Angelo C, Frana J, Montico S, Carrancio L, Todelo C, Saluso A. 2018. Ordenación territorial participativa en espacios periurbanos: La problemática de las aplicaciones de fitosanitarios. En: Titonell P y Giobellina B, Periurbanos hacia el Consenso. Libro 1. Ediciones INTA, 674 pp. https://repositorio.inta. gob.ar/xmlui/handle/20.500.12123/3035

Fernández Equiza AM (compiladora). 2017. Debates sobre naturaleza y desarrollo: análisis a distintas escalas. 1ra. Edición, Universidad Nacional del Centro de la Prov. De Buenos Aires, Tandil. Libro digital: http://biblioteca. clacso.edu.ar/clacso/se/20180515030535/ Debates_naturaleza_desarrollo.pdf

Fior M, Guglielmone P. 2017. Abordar la comunicación desde la complejidad. En: Milo Vaccaro M y Aradas Diaz ME. Revista Construyendo Vínculos 2. Ediciones INTA EEA OLIVEROS. 120 pp. https://inta.gob.ar/sites/default/files/ inta.revista-construyendo-vinculos.pdf

García R. 2006. Sistemas complejos: Conceptos, Método y Fundamentación. Editorial GEDISA. Barcelona. 200 pp.

Longo A, Carrancio L, Gerster G, Timoni R, Cardozo F, Mondino MC, Ibarlucea J, Morlacco MB, Correa Luna M, Bertozzi E, Gadda A, Casella E. 2017. Elaboración de catálogo de modos productivos (ECOM/INTA). Periurbano hacia el consenso. Ciudad Ambiente y Producción de Alimentos. $1^{\circ}$ Encuentro Nacional de Periurbanos e Inter-fases Criticas. $2^{\circ}$ reunión Científica de PNNAT. $3^{\circ}$ Reunión de la Red PERIURBAN. Córdoba. Argentina
Morello J. 2000. Funciones del sistema periurbano: el caso de Buenos Aires. Mar del Plata: Universidad Nacional de Mar del Plata-Ediciones CIAM/GADU.

Pretlove B, Blasiak R. 2018. Mapping Ocean Governance and Regulation. Working paper for consultation for UN Global Compact Action Platform for Sustainable Ocean Business. Disponible en: https://www.researchgate.net/ publication/327884976_Mapping_Ocean_ Governance_and_Regulation/download

Propersi P, Albanessi R, Tifni E, Pérez M, Pérez R, Galetto M, Mezzo R, Aradas Díaz ME. 2017. Relevamiento de sistemas productivos del área periurbana de la localidad de San Genaro Santa Fe. En: Milo Vaccaro M y Aradas Diaz ME. Revista Construyendo Vínculos 2. Ediciones INTA EEA OLIVEROS. 120 pp. https://inta.gob.ar/sites/default/files/ inta.revista-construyendo-vinculos.pdf

Zulaica L, Ferraro R. 2011. Aportes metodológicos para la gestión territorial del periurbano marplatense (Provincia de Buenos Aires, Argentina): Aplicaciones en el sector sur. Revista Geográfica de América Central. Número Especial EGAL, 2011- Costa Rica. II Semestre 2011, pp. 1-21. 